\title{
HPLC Fingerprints for the Authentication of Cranberry-Based Products Based on Multivariate Calibration Approaches
}

\author{
Lidia Puigventós ${ }^{\text {a }}$, Oscar Núñez ${ }^{*, a, b, c}$, Javier Saurinaa,b \\ ${ }^{a}$ Department of Analytical Chemistry, University of Barcelona. Martí i Franquès 1-11, E-08028, Barcelona, \\ Spain. ${ }^{b}$ Research Institute in Food Nutrition and Food Safety, University of Barcelona, Recinte Torribera, \\ Av. Prat de la Riba 171, Edifici de Recerca (Gaudí), E-08921 Santa Coloma de Gramenet, Barcelona, Spain. \\ 'Serra Hunter Fellow, Generalitat de Catalunya, Barcelona, Spain.
}

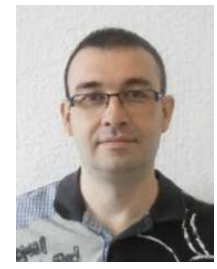

\begin{abstract}
This work introduces the topic of the authentication of cranberry-based products and the detection and quantification of possible adulterations with other raw materials of lower quality. For such a purpose, genuine and adulterated cranberry samples were analyzed by reversed-phase HPLC with UV detection. Sample components were separated using an elution gradient based on $0.1 \%(v / v)$ formic acid aqueous solution and methanol as the components of the mobile phase. Chromatograms were recorded at 280, 370 and $520 \mathrm{~nm}$. Data resulting from the injection of pure and adulterated samples, consisting of chromatographic fingerprints at each detection wavelength, were analyzed chemometrically. Preliminary studies by Principal Component Analysis showed that the sample extracts were clearly distributed depending on the extent of adulteration. Data was further treated by Partial Least Square regression to determine the percentages of grape contamination. It was found that even mixture samples containing low percentages of grape could be distinguished from genuine cranberry extracts. Besides, results obtained were highly satisfactory, with overall quantification errors lower than 5\%. As a conclusion, the method proposed here resulted in an excellent approach to carry out the authentication of cranberry-based products relying on polyphenolic fingerprints.
\end{abstract}

Keywords: Liquid chromatography, polyphenolic fingerprints, cranberry, authentication, chemometrics, Partial Least Square regression

\section{INTRODUCTION}

Food products and pharmaceuticals prepared with American red cranberries (Vaccinium macrocarpon) are gaining importance in our society due to some healthy effects on humans, including antioxidant, anti-inflammatory and bacteriostatic activities [1]. Although some of these properties have been attributed to the occurrence of high levels of overall polyphenols, antibiotic effects are quite specific of A-type proanthocyanidins (PACs), a type of flavonoid unique of cranberries [2,3]. Nowadays, however, some concerns have arisen on the evaluation of authenticity of red cranberry extracts as well as on the detection of possible adulterations with less expensive fruits like grapes or blueberries, which do not contain the appropriate PACs for having the desired bioactivity. This fact reveals the importance of developing analytical methodologies for the characterization of natural extracts to deal with authentication issues regarding the fruit of origin.

Cranberries contain high amounts of polyphenols including benzoic and cinnamic acids, and flavonoids such as anthocyanins, flavonols, and flavan-3-ols [4,5]. In particular, flavan-3-ols (catechins and epicatechins) occur in cranberry in both monomeric and polymeric forms (i.e., PACs).

*Address correspondence to this author at the Department of Analytical Chemistry, Faculty of Chemistry, University of Barcelona, Martí i Franquès 1-11, E-08028, Barcelona, Spain; Tel/Fax: +34-93-4033706, +34-934021233; E-mails: oscar.nunez@ub.edu; oscarnubu@yahoo.es
PACs are often classified according to the interflavan linkage as A-type and B-type molecules. B-type PACs are those in which monomeric units are linked through the $\mathrm{C} 4$ position of the upper unit and the $\mathrm{C} 6$ or $\mathrm{C} 8$ positions of the lower unit. A-type PACs contain an additional ether-type bond between the $\mathrm{C} 2$ position of the upper unit and the hydroxyl group at $\mathrm{C} 7$ or $\mathrm{C} 5$ positions of the lower unit (C2$\mathrm{O}-\mathrm{C} 7$ or C2-O-C5) (see scheme in Figure 1). In general, 60\% of PACs in cranberry are A-type ones [6]. B-type PACs are found in other food products like tea, chocolate, blueberry or grapes.

Various analytical methods have been proposed to tackle the determination of PACs in fruit products. A rough estimation of overall contents can be assessed by simple colorimetric analysis based on the measurement of the absorption at $545 \mathrm{~nm}$. A more sensitive assay rely on the reaction of PACs with 4-dimethylaminocinnamaldehyde (DMAC) [7,8]. Obviously, these methods are not selective and just provide an overall PAC index unable to differentiate among A-, B-types and other related molecules. For a more comprehensive insight in the PAC analysis, liquid chromatography (HPLC) with UV detection or coupled to mass spectrometry (LC-MS) [9-12] have been proposed. Analytes are typically separated by reversed-phase mode using acidified aqueous solutions and methanol or acetonitrile as the components of the mobile phase [13]. In general, the great structural diversity of PACs due to the variety of polymerization possibilities [14] leads to complex profiles with multiple peak overlapping. In this regard, high 
resolution mass spectrometry (HRMS) may contribute to achieve a more efficient characterization of such complex polyphenols [15]. As an example, Rockenbach et al. [16] used a Fourier-transform ion cyclotron resonance mass spectrometry (FTICR-MS) being able to characterize and assign the elemental composition of 251 different flavan-3-ol compounds in grapes. Regarding cranberry-based products, Iswaldi et al. proposed the use of time-of-flight mass spectrometry (TOF-MS) for the characterization of the phenolic fraction in cranberry syrup [11]. Apart from chromatography, capillary electrophoresis (CE) has appeared in the analytical arena as an alternative technique for the determination of polyphenolic compounds in fruit-based products [17-21].

Characterization and classification of fruit products can be tackled from compositional profiles as a source of analytical information. For such a purpose, polyphenols as well as other low molecular weight organic molecules can be used as taxonomical markers to infer the origin of fruits. In this context, chemometric methods have been exploited to facilitate the extraction of relevant information from the analysis of compositional profiles or complex instrumental fingerprints of the corresponding samples [22]. Principal component analysis (PCA) is commonly focused on exploratory studies of characterization and classification of food products. More specific research on classification and pattern recognition can be conducted by discriminant analysis (DA), partial least squares-discriminant analysis (PLS-DA) and soft independent analysis of class analogies (SIMCA). For quantitative purposes, PLS is the one of the most popular methods for dealing with the determination of adulteration percentages.

In a previous publication, a preliminary characterization and discrimination of cranberry- and grapebased products was performed [23]. The method was based on fruit lyophilization to get dry fruit products, liquid extraction and purification to recover the polyphenolic fraction, and chromatographic analysis of extracts by HPLC and CE. Separation profiles were analyzed chemometrically and some relevant descriptors related to polyphenols, including PACs, were encountered. However, in this last method only $100 \%$ cranberry-based and grape-based samples were analyzed. The present work represents a step forward towards the detection and quantification of adulterations of cranberry extracts with grape-based products. For such a purpose, genuine cranberry and grape samples as well as extract mixtures of the two fruits (containing grape concentrations in the range 2.5 to $50 \%$ ) have been analyzed by reversed-phase HPLC-UV. Chromatographic data registered at 280, 370 and $520 \mathrm{~nm}$ have been used as a source of analytical information that potentially contain descriptors of fruit products. PCA has been applied to carry out a preliminary evaluation of the suitability of the different sets of data and pretreatments applied. A further study by PLS has been conducted to quantify the percentage of grape extracts present in the samples. PLS results have proved that the proposed method is suitable to detect adulteration of cranberry extracts. Results have been considered as the basis of further comprehensive authentication studies of great interest in food control to prevent misuses.

\section{EXPERIMENTAL}

\subsection{Chemicals and standards}

Unless specified, analytical grade reagents were used. The mobile phase was prepared with Milli-Q water (Millipore, Milford, MA), formic acid ( $99 \% \mathrm{w} / \mathrm{w}$, analytical grade, from Merck, Darmstadt, Germany) and methanol (MeOH, from Panreac, Barcelona, HPLC grade).

\subsection{Samples}

Cranberries and grapes (fresh fruits and raisins) were purchased from Barcelona markets. Prior to sample treatment, fruits and raisins were lyophilized to obtain a powdered product similar to natural or commercial pharmaceutical samples. For that purpose, samples remained 24 hours inside a lyophilizer (Lioalfa-6, Telstar, Terrassa, Spain) from $-80{ }^{\circ} \mathrm{C}$ to room temperature and after that fruit products rested $6.5 \mathrm{~h}$ at $40^{\circ} \mathrm{C}$.

Sample treatment was carried out following a previously described method [24]. Briefly, $0.1 \mathrm{~g}$ of sample were dispersed in $10 \mathrm{~mL}$ of acetone: $\mathrm{H}_{2} \mathrm{O}: \mathrm{HCl}(70: 29.9: 0.1$ $v / v / v)$ and sonicated for $30 \mathrm{~min}$. The mixture was then centrifuged for $15 \mathrm{~min}$ at $3500 \mathrm{rpm}$, and the extracts were stored at $-4^{\circ} \mathrm{C}$ until analyzed. Before injection, extracts were filtered through $0.45 \mu \mathrm{m}$ nylon filters (Whatman, Clifton, NJ, USA).

5 cranberry sample extracts and 5 grape sample extracts were processed as indicated above. Apart from the corresponding pure working solutions of cranberry and grape, mixtures with different percentages of grape as the adulterant were prepared, namely: $50 \%$ grape (5 samples), $10 \%$ grape (3 samples), $7.5 \%$ grape ( 5 samples), $5 \%$ grape (3 samples) and $2.5 \%$ grape (5 samples).

\subsection{Liquid chromatographic method}

The chromatographic system consisted of Agilent 1100 Series HPLC instrument equipped with a G1311A quaternary pump, a G1379A degasser, a G1392A autosampler, a G1315B diode-array detector and a PC with the Agilent Chemstation software (Rev. A 10.02), all of them from Agilent Technologies (Waldbronn, Germany). The separation column was a Kinetex C18 $(100 \mathrm{~mm} \times 4.6 \mathrm{~mm}$ i.d., particle size $2.6 \mu \mathrm{m}$ ) furnished with a SecurityGuard C18 cartridge (both from Phenomenex, Torrance, CA). The separation was based on the following gradient using $0.1 \%$ $(\mathrm{v} / \mathrm{v})$ formic acid aqueous solution and $\mathrm{MeOH}$ as the components of the mobile phase: 0 to $3 \mathrm{~min}$, linear gradient from $5 \% \mathrm{~B}$ (initial conditions) to $25 \% \mathrm{~B} ; 3$ to $6 \mathrm{~min}$ isocratic step at $25 \% \mathrm{~B} ; 6$ to 9 min linear gradient from $25 \%$ B to $37 \%$ $\mathrm{B} ; 9$ to $13 \mathrm{~min}$ isocratic step at $37 \% \mathrm{~B}$; $13-18$ min linear gradient from $37 \% \mathrm{~B}$ to $54 \% \mathrm{~B} ; 18-22 \mathrm{~min}$ isocratic step at $54 \%$ B; 22-26 min linear gradient from $54 \%$ B to $95 \%$ B; 2629 min isocratic step at $95 \% \mathrm{~B} ; 29$ to 29.15 min back to initial conditions at $5 \% \mathrm{~B}$ and from 29.15 to $36 \mathrm{~min}$ isocratic step at initial conditions at $5 \% \mathrm{~B}$. The flow rate was $1 \mathrm{~mL}$ $\min ^{-1}$ and the injection volume $10 \mu \mathrm{L}$. Chromatograms were recorded at 280, 370 and $520 \mathrm{~nm}$.

\subsection{Data processing and analysis}

Chromatograms were pretreated as follows to improve the data quality while minimizing solvent and matrix interferences, peak shifting and baseline drifts. The working 
time range was selected after removing the time windows 0 to $2.5 \mathrm{~min}$ and 25 to $37 \mathrm{~min}$ as they corresponded to the dead volume front and cleaning step. Chromatograms from different sample injections were synchronized using various representative peaks distributed throughout the chromatogram as references. Baseline offsets were also corrected by repositioning to zero the absorbance of the initial time channel of each window. For additional details see ref. 25.

Stand Alone Chemometrics Software (SOLO) from Eigenvector Research was used for PCA and PLS calculations [26]. A detailed description of theoretical background of these methods is given elsewhere [27].

For exploratory studies by PCA, data matrices were built from chromatographic profiles recoded for the set of samples under study. In all the cases, data was autoscaled to provide similar weights to all variables. Scatter plots of scores and loadings of the principal components (PCs) were used to investigate the structure of maps of samples and variables, respectively.

The quantification of the percentage of grape extract in the samples was based on PLS. Samples available were distributed among training and test sets as follows. Training set: $100 \%$ grape (3 samples), $50 \%$ grape (3 samples), $10 \%$ grape (2 samples), $7.5 \%$ grape ( 3 samples), $5 \%$ grape (2 samples), $2.5 \%$ grape (3 samples) and $100 \%$ cranberry (3 samples). The remaining samples considered as unknown were used for validation and prediction purposes. For both training and test steps, X-data matrices consisted of the chromatographic profiles of the corresponding matrices and the Y-data matrices contained the grape percentages.

\section{RESULTS AND DISCUSSION}

\subsection{Principal component analysis}

First, some preliminary HPLC-UV analyses of pure cranberry-based and grape-based extracts as well as cranberry samples adulterated with grape extracts were performed. As an example, Figure 2 compares the chromatograms of representative cranberry and grape samples. It can be seen that the intensity of cranberry signals is about 10-fold higher (or even more) than that of the grape samples. Furthermore, the chromatogram of cranberry is much richer in peaks and, thus, in the diversity of compounds. For this reason, the chromatogram of cranberry samples adulterated with $10 \%$ grape was very similar to that of the pure cranberry (see Figure 2). Anyway, when focusing our attention on selected time windows some characteristic features of grapes can be observed. The intensity of these features is low so that they appeared imbibed below the greater contributions of cranberry components. This finding suggests that the detection of the addition of low percentages of grape as adulterant of cranberries will be difficult as characteristic grape peaks are minor and scarce.

Preliminary PCA characterization was attempted using raw chromatograms at 280, 370 and $520 \mathrm{~nm}$ as the analytical data (see representative chromatograms of a cranberry sample and a grape sample in Figure 3). The performance of the resulting models was quite limited due to experimental variability (e.g., peak shifting and baseline correction) that strongly affected the distribution of samples. It was thus concluded that chromatogram preprocessing was recommendable to enhance the analytical performance of PCA models.

For that purpose, data was treated subsequently as indicated in the experimental section to obtain the corrected chromatograms. PCA on these data sets indicated that samples were much more structured according to the grape percentages so results were studied more thoroughly as follows. In the case of chromatograms at $520 \mathrm{~nm}$, despite the occurrence of some colored components absorbing at this wavelength, the magnitude of the corresponding peaks was low (see Figure 3) so that the discrimination performance was poor.

At $280 \mathrm{~nm}$, conversely, chromatograms displayed multiple high intense peaks basically attributed to the polyphenolic families of benzoic and cinnamic acids (Figure 3 ). Unfortunately, the strong peak overlapping due to the coelution of intense absorbing compounds hindered the extraction of robust conclusions. As an additional drawback, the contribution of characteristic grape components to the signal fingerprints was much lower than that attributed to cranberry species. In this way, although samples with high grape percentages (50\% grape) appeared far away from pure cranberries, those with grape contents lower that $10 \%$ were hardly distinguished from unaltered cranberry extracts.

Chromatograms recorded at $370 \mathrm{~nm}$ (Figure 3) reflected the diversity of components belonging to flavonoid families including, for instance, monomeric and polymeric (PAC) catechins. In contrast, multiple peak coming from phenolic acids were avoided. In this case, chromatograms were simpler than those recorded at $280 \mathrm{~nm}$ and peaks were better resolved. Besides, various small features quite characteristic of grapes were detected so that we believed that the discriminant capacity of this set could be better (especially regarding PAC components).

Figure 4 shows the scatter plot of scores of PC1 versus PC2. Samples with similar grape percentage were grouped in close positions. PC1 described the sample distribution as a function of cranberry content, with pure cranberry extracts at the right and the most contaminated ones (50\% grape) at the left. Accordingly, those mixtures with low grape amounts were found next to the genuine cranberry cluster. PC2 discriminated among pure grape (top) and cranberrycontaining samples. The study of loadings revealed the most significant variables governing study. Characteristic peaks of cranberries had high loading values on PC1 while peaks of grape components displayed high levels on PC2. This exploratory analysis PCA allow us to propose polyphenolic chromatographic profiles recorded at $370 \mathrm{~nm}$ as the best data-set to carry out further studies.

\subsection{Partial least squares regression}

PLS was applied to quantify the grape percentage of adulteration in the cranberry sample extracts under study. The PLS model was first established on the data set of calibration as indicated in the experimental section. Results discussed here corresponded to data recorded at $370 \mathrm{~nm}$. It should be mentioned that models using chromatograms recorded at 280 and $520 \mathrm{~nm}$ were also investigated but, again, the most satisfactory PLS results were obtained at 370 $\mathrm{nm}$. The number of latent variables (LV) to be used for the assessment of the model was estimated by cross validation 
on random sets, considering 4 data splits and 20 iterations. As shown in Figure $5 \mathrm{~b}$ the lowest prediction error was attained with $4 \mathrm{LV}$ so this number was chosen for further studies. Figure $5 \mathrm{~b}$ depicts the scatter plot of scores on LV1 and LV2. The distribution of samples was in agreement with the grape contents, with pure grape extracts on the top left corner and genuine and low adulterated cranberry extracts on the bottom right corner. The performance of predictions of grape percentages in both calibration and prediction steps was evaluated under the selected model conditions. Training and test results have been summarized in Figures $5 \mathrm{c}$ and $5 \mathrm{~d}$, respectively. It can be seen that the agreement between actual and predicted values was highly satisfactory. In the case of the test set, overall prediction errors were below $5 \%$. Therefore, it was concluded that the method was suitable to quantify the percentage of grapes as adulteration matter of cranberry samples.

\section{CONCLUSION}

In this work, we have demonstrated the suitability of chromatographic fingerprints as the source of data to carry out the study of the authentication of cranberry samples. The percentage of grape added as adulterant to the cranberry extracts can be determined by multivariate calibration using partial least squares regression. Data preprocessing, especially peak alignment to minimize the feature variability among chromatograms, has shown to be essential to obtain suitable calibration models and accurate predictions. From the chemometric point of view, the complexity of the analytical problem to be solved arises in the fact that the number (and intensity) of characteristic features of grape signals is much lower than that of cranberry and, in consequence, the detection of low amounts of grape is envisaged as a difficult issue. Despite the poor selectivity and sensitivity of grape fingerprints, chromatograms at $370 \mathrm{~nm}$, which display polyphenolic features mainly associated to single flavonoids and polymeric catechins, show some characteristic grape peaks. Indeed, these features results in the basis to tackle the grape quantification. Behind these particular results, we guess that the strategy proposed here may be extended to other food adulteration cases and sample authentication could be conducted in a similar way.

\section{LIST OF ABBREVIATIONS}

CE, Capillary electrophoresis

DMAC, 4-dimethylaminocinnamaldehyde

FTICR-MS, Fourier-transform ion cyclotron resonance mass spectrometry

HPLC, High performance liquid chromatography

HRMS, High resolution mass spectrometry

LC-MS, Liquid chromatography coupled to mass spectrometry

PAC, Proanthocyanidins

PCA, Principal component analysis

PLS, Partial least squares (regression)

PLS-DA, Partial least squares-discriminant analysis

TOF-MS, Time-of-flight mass spectrometry

\section{UV, Ultraviolet}

\section{CONFLICT OF INTEREST}

This work has been funded by the Spanish Ministry of Economy and Competitiveness under the projects CTQ2012-
30836 and CTQ2014-65324, and from the Agency for Administration of University and Research Grants (Generalitat de Catalunya, Spain) under the projects 2014 SGR-377 and 2014 SGR-539.

Authors declare that we do not have any conflict of interest.

\section{REFERENCES}

[1] Sanchez-Patan, F.; Bartolome, B.; Martin-Alvarez, P.J.; Anderson, M.; Howell, A.; Monagas, M. Comprehensive Assessment of the Quality of Commercial Cranberry Products. Phenolic Characterization and in Vitro Bioactivity. J. Agric. Food Chem. 2012, 60, 3396-3408.

[2] Foo L.Y., Lu Y., Howell A.B., Vorsa N. The structure of cranberry proanthocyanidins which inhibit adherence of uropathogenic Pfimbriated Escherichia coli in vitro. Phytochemistry 2000, 54, 173 181.

[3] Howell A.B., Reed J.D., Krueger C.G., Winterbottom R., Cunningham D.G., Leahy M. A-type cranberry proanthocyanidins and uropathogenic bacterial anti-adhesion activity. Phytochemistry 2005, 66, 2281-2291

[4] Gu L., Kelm M.A., Hammerstone J.F., Beecher G., Holden J. Haytowitz D., Prior R.L. Screening of Foods Containing Proanthocyanidins and Their Structural Characterization Using LCMS/MS and Thiolytic Degradation. J. Agric. Food Chem. 2003, 51, 7513-7521.

[5] Guyot, S. Flavan-3-Ols and Proanthocyanidins, in Nollet, N.M.L \& Toldrá, F. (eds.), Handbook of Analysis of Active Compounds in Functional Foods, CRC Press, Boca Raton, FL, USA, 2012.

[6] Gu L., Kelm M.A., Hammerstone J.F., Beecher G., Holden J., Haytowitz D., Gebhardt S., Prior R.L. Concentrations of proanthocyanidins in common foods and estimations of normal consumption. J. Nutr. 2004, 134, 613-617.

[7] Feliciano, R.P.; Shea, M.P.; Shanmuganayagam, D.; Krueger, C.G.; Howell, A.B.; Reed, J.D. Comparison of Isolated Cranberry (Vaccinium macrocarpon Ait.) Proanthocyanidins to Catechin and Procyanidins A2 and B2 for Use as Standards in the 4(Dimethylamino)cinnamaldehyde Assay. J. Agric. Food Chem. 2012, 60, 4578-4585.

[8] Prior, R.L.; Fan, E.; Ji, H.; Howell, A.; Nio, C.; Payne, M.J.; Reed, J. Multi-laboratory validation of a standard method for quantifying proanthocyanidins in cranberry powders. J. Sci. Food Agric. 2010, 90, 1473-1478.

[9] Motilva, M.J.; Serra, A.; Macia, A. Analysis of food polyphenols by ultra high-performance liquid chromatography coupled to mass spectrometry: An overview, J. Chromatogr. A 2013, 1292, 66-82.

[10] Appeldoorn, M.M.; Vincken, J.P.; Sanders, M.; Hollman, P.C.H.; Gruppen, H. Combined Normal-Phase and Reversed-Phase Liquid Chromatography/ESI-MS as a Tool To Determine the Molecular Diversity of A-type Procyanidins in Peanut Skins. J. Agric. Food Chem. 2009, 57, 6007-6013.

[11] Iswaldi, I.; Gomez-Caravaca, A.M.; Arraez-Roman, D.; Uberos, J.; Lardon, M.; Segura-Carretero, A.; Fernandez-Gutierrez, A. Characterization by high-performance liquid chromatography with diode-array detection coupled to time-of-flight mass spectrometry of the phenolic fraction in a cranberry syrup used to prevent urinary tract diseases, together with a study of its antibacterial activity. J. Pharm. Biomed. Anal. 2012, 58, 34-41.

[12] Wallace, T.C.; Giusti, M.M. Extraction and normal-phase HPLCfluorescence-electrospray MS characterization and quantification of procyanidins in cranberry extracts. J. Food Sci. 2010, 75, C690C696.,

[13] Saurina, J.; Sentellas, S. Determination of Phenolic Compounds in Food Matrices: Applications to Characterization and Authentication, Chapter 13, in: Núñez, O.; Gallart-Ayala, H. Martins, C.P.B.; Lucci P. (Eds), Fast Liquid Chromatography-Mass Spectrometry Methods in Food and Environmental Analysis, Imperial College Press, London, p.p. 517-547, 2015.

[14] Patel, K.D.; Scarano, F.J.; Kondo, M.; Hurta, R.A.R.; Neto, C.C. Proanthocyanidin-rich extracts from cranberry fruit (Vaccinium macrocarpon Ait.) selectively inhibit the growth of human pathogenic fungi Candida spp. and Cryptococcus neoformans. J. Agric. Food Chem. 2011, 59, 12864-12873.

[15] P. Lucci, C.P.B. Martins. Liquid Chromatography-High Resolution Mass Spectrometry in Environmental and Food Analysis, Chapter 8, in: Núñez, O.; Gallart-Ayala, H.; Martins, C.P.B.; Lucci P. (Eds), Fast Liquid Chromatography-Mass 
Spectrometry Methods in Food and Environmental Analysis, Imperial College Press, London, p. 325-345, 2015.

[16] Rockenbach, I.I.; Jungfer, E.; Ritter, C.; Santiago-Schuebel, B.; Thiele, B.; Fett, R.; Galensa, R. Characterization of flavan-3-ols in seeds of grape pomace by CE, HPLC-DAD-MSn and LC-ESIFTICR-MS. Food Res. Int. 2012, 48, 848-855.

[17] Sawalha, S.M.S.; Arraez-Roman, D.; Segura-Carretero, A.; Fernandez-Gutierrez, A. Quantification of main phenolic compounds in sweet and bitter orange peel using CE-MS/MS. Food Chem. 2009, 116, 567-574.

[18] Helmja, K.; Vaher, M.; Puessa, T.; Kaljurand, M. Analysis of the stable free radical scavenging capability of artificial polyphenol mixtures and plant extracts by capillary electrophoresis and liquid chromatography-diode array detection-tandem mass spectrometry. J. Chromatogr. A 2009, 1216, 2417-2423.

[19] Peres, R.G.; Micke, G.A.; Tavares, M.F.M.; Rodriguez-Amaya, D.B. Multivariant optimization, validation, and application of capillary electrophoresis for simultaneous determination of polyphenols and phenolic acids in Brazilian wines. J. Sep. Sci. 2009, 32, 3822-3828.

[20] Franquet-Griell, H.; Checa, A.; Núñez, O.; Saurina, J.; HernandezCassou, S.; Puignou, L. Determination of Polyphenols in Spanish Wines by Capillary Zone Electrophoresis. Application to Wine Characterization by Using Chemometrics. J. Agric. Food Chem. 2012, 60, 8340-8349.

[21] Carrasco-Pancorbo, A.; Gomez-Caravaca, A.M.; Segura-Carretero, A ; Cerretani, L.; Bendini, A.; Fernandez-Gutierrez, A. Use of capillary electrophoresis with UV detection to compare the phenolic profiles of extra-virgin olive oils belonging to Spanish and Italian PDOs and their relation to sensorial properties. J. Sci. Food Agric. 2009 , $89,2144-2155$

[22] Saurina, J. Characterization of wines using compositional profiles and chemometrics, Trends Anal. Chem. 2010, 29, 234-245.

[23] Navarro, M.; Núñez, O.; Saurina, J.; Hernández-Cassou, S.; Puignou, L. Characterization of fruit products by capillary zone electrophoresis and liquid chromatography using the compositional profiles of polyphenols: Application to authentication of natural extracts, J. Agric. Food Chem. 2014, 62, 1038-1046.

[24] Puigventós, L; Navarro, M; Alechaga, E; Núñez, O; Saurina, J Hernández-Cassou, S.; Puignou, L. Determination of polyphenolic profiles by liquid chromatography-electrospray-tandem mass spectrometry for the authentication of fruit extracts, Anal. Bioanal. Chem. 2015, 407, 597-608.

[25] Perez-Rafols, C.; Saurina, J. Liquid chromatographic fingerprints and profiles of polyphenolic compounds applied to the chemometric characterization and classification of beers, Anal. Methods, 2015 , 7, 8733-8733.

[26] http://www.eigenvector.com/software/solo.htm

[27] Massart, D.L.; Vandeginste, B.G.M.; Buydens, L.M.C.; de Jong, S.; Lewi, P.J.; Smeyers-Verbeke, J. Handbook of Chemometrics and Qualimetrics, Elsevier, Amsterdam, 1997. 
Figure 1. Chemical structure of A- and B-type interflavan links of proanthocyanidins.

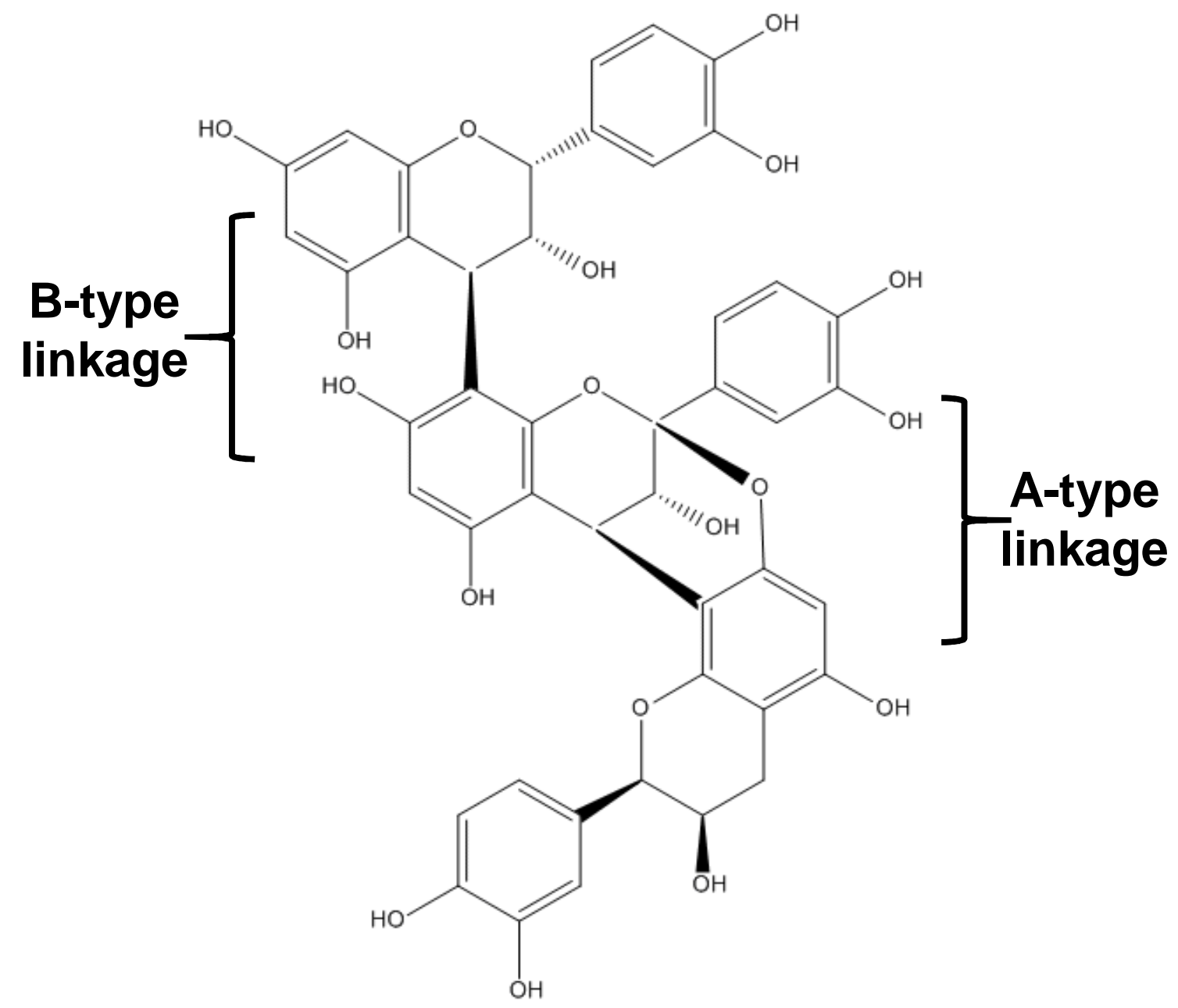


Figure 2. Chromatograms of cranberry, grape and mixture $(90 \%$ cranberry $10 \%$ grape) samples at $280 \mathrm{~nm}$.

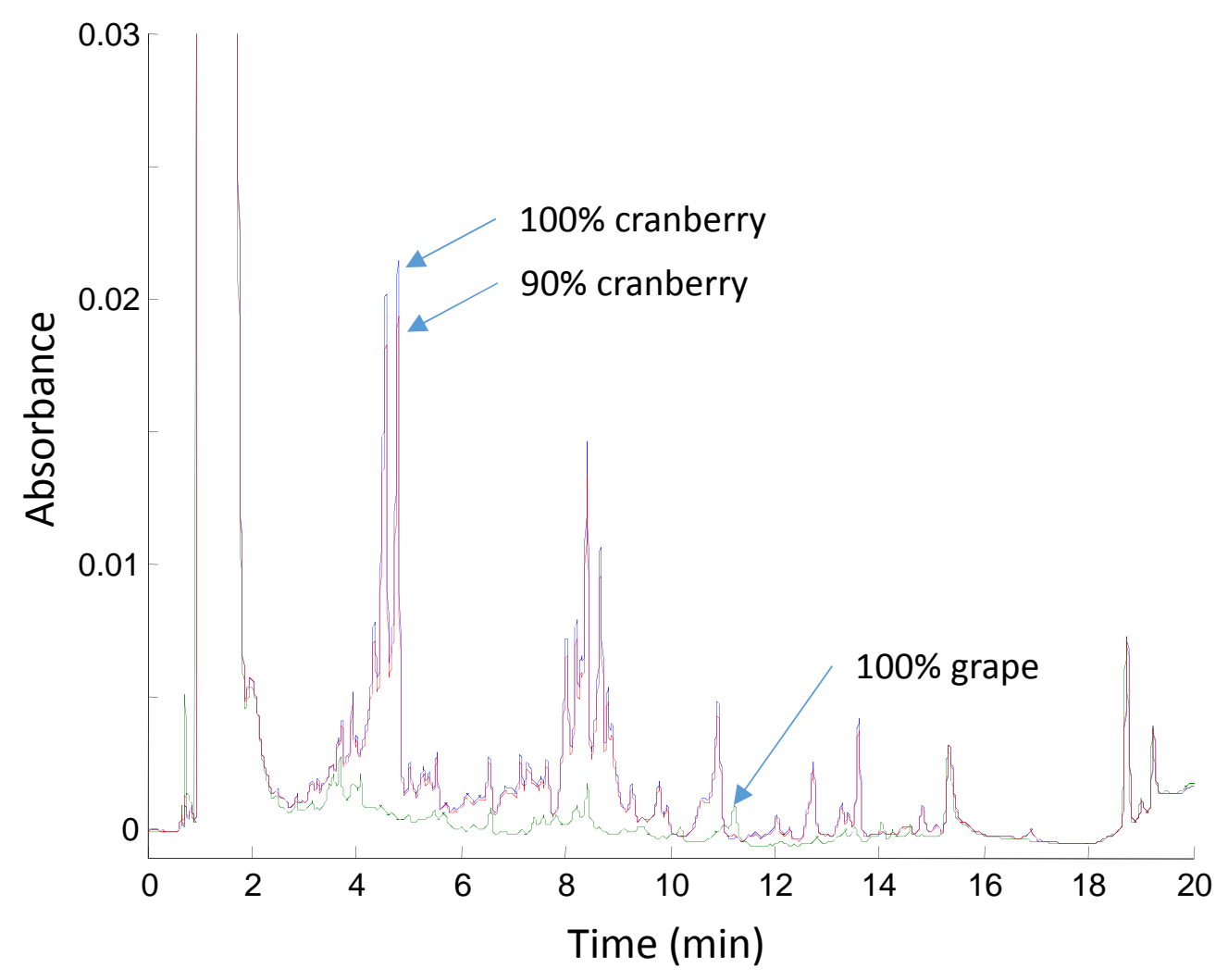


Figure 3. Chromatograms of cranberry and grape samples recorded at 280, 370 and $520 \mathrm{~nm}$ representing fingerprints of phenolic acid, flavonoid and anthocyanin components, respectively.

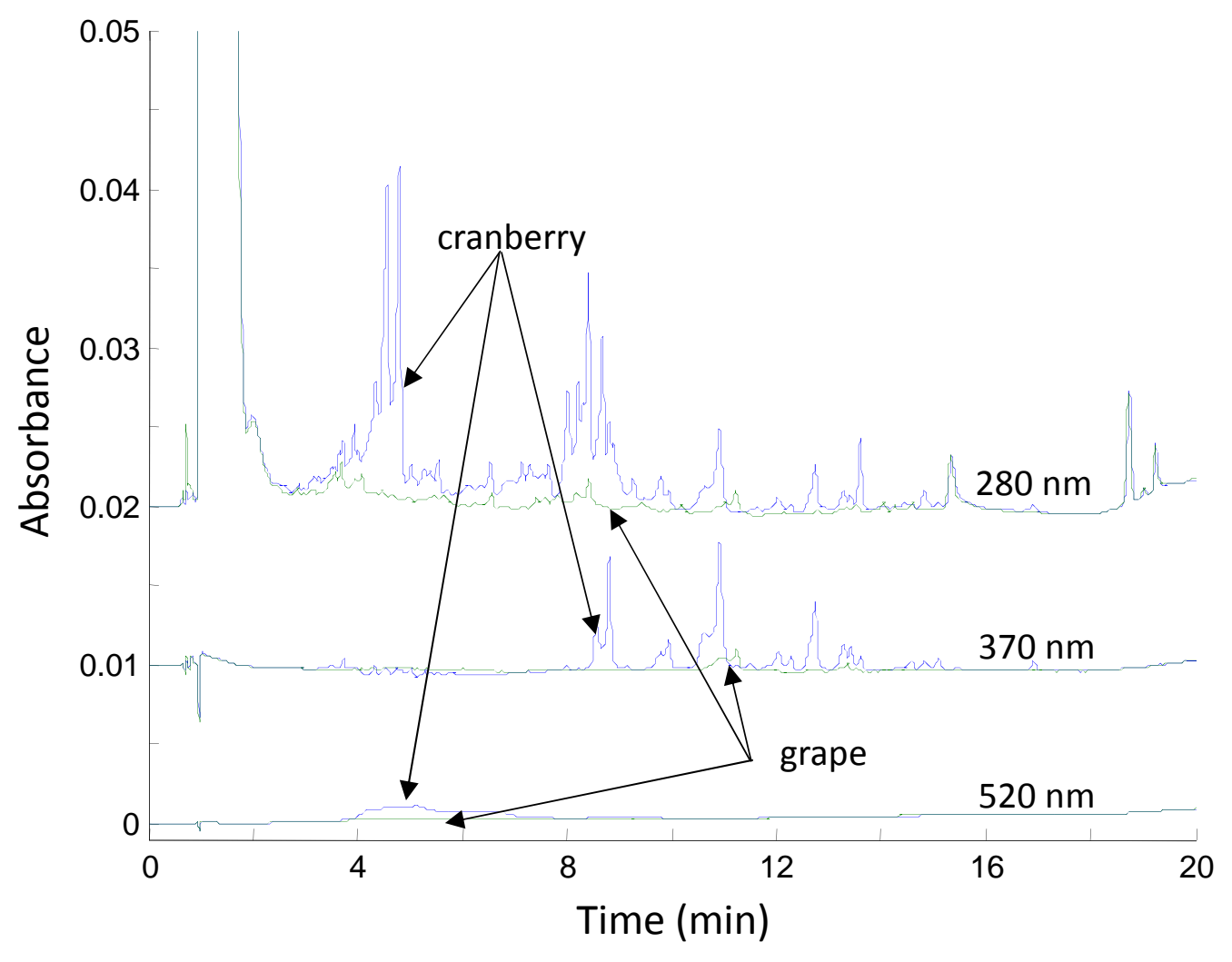


Figure 4. Plot of scores by principal component analysis using the chromatographic fingerprints taken at $370 \mathrm{~nm}$ as the data set.

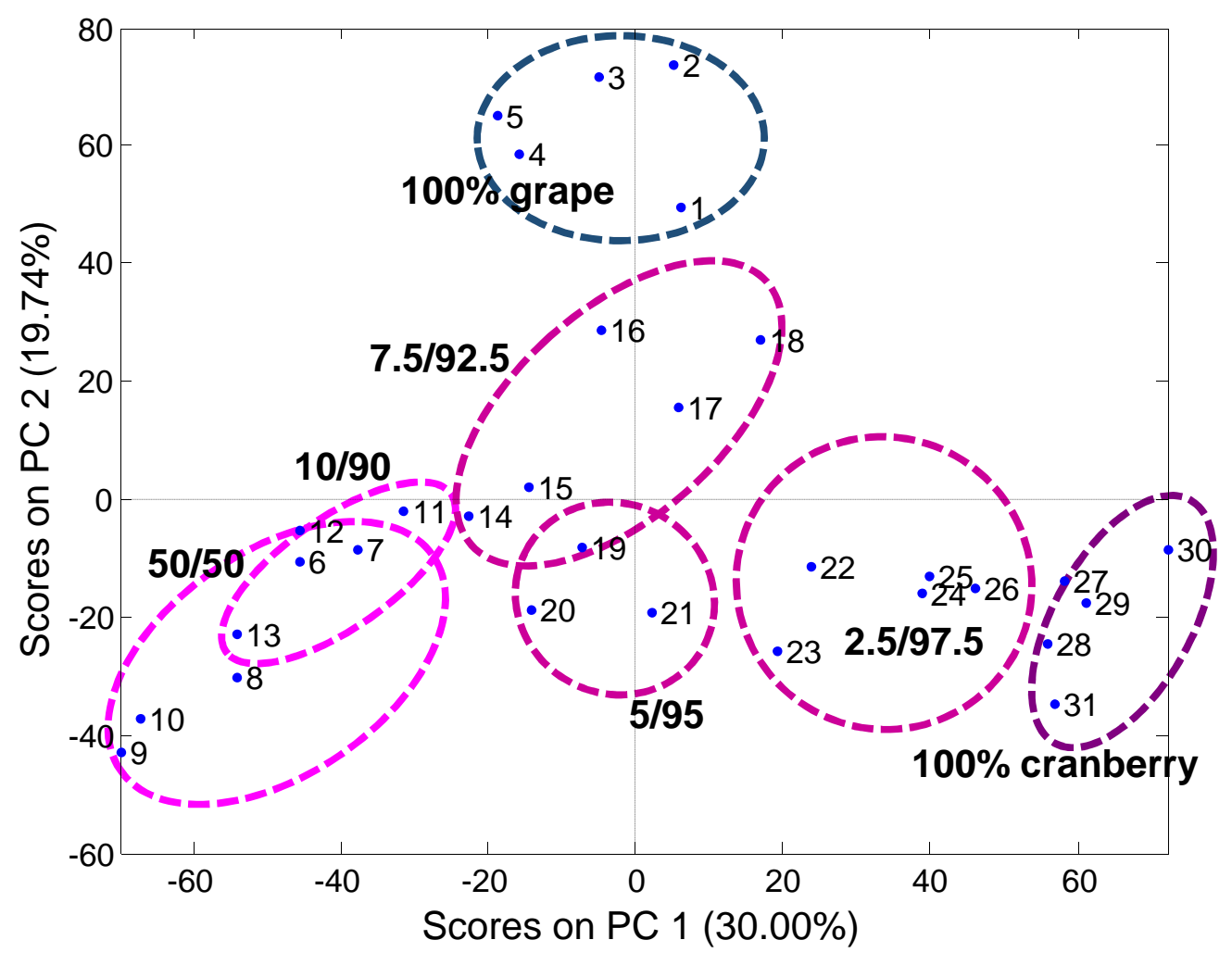


Figure 5. Partial least square regression applied to the quantification of the grape percentage of the samples. (a) Plot of scores of latent variable 1 versus latent variable 2. (b) Root mean square error in cross validation (RMSECV) for the estimation of the optimum number of latent variables to be used for the assessment of the calibration model. (c) Scatter plot of actual vs calculated grape percentages in the validation of the calibration model. (d) Scatter plot of actual vs calculated grape percentages in the validation of predictions.
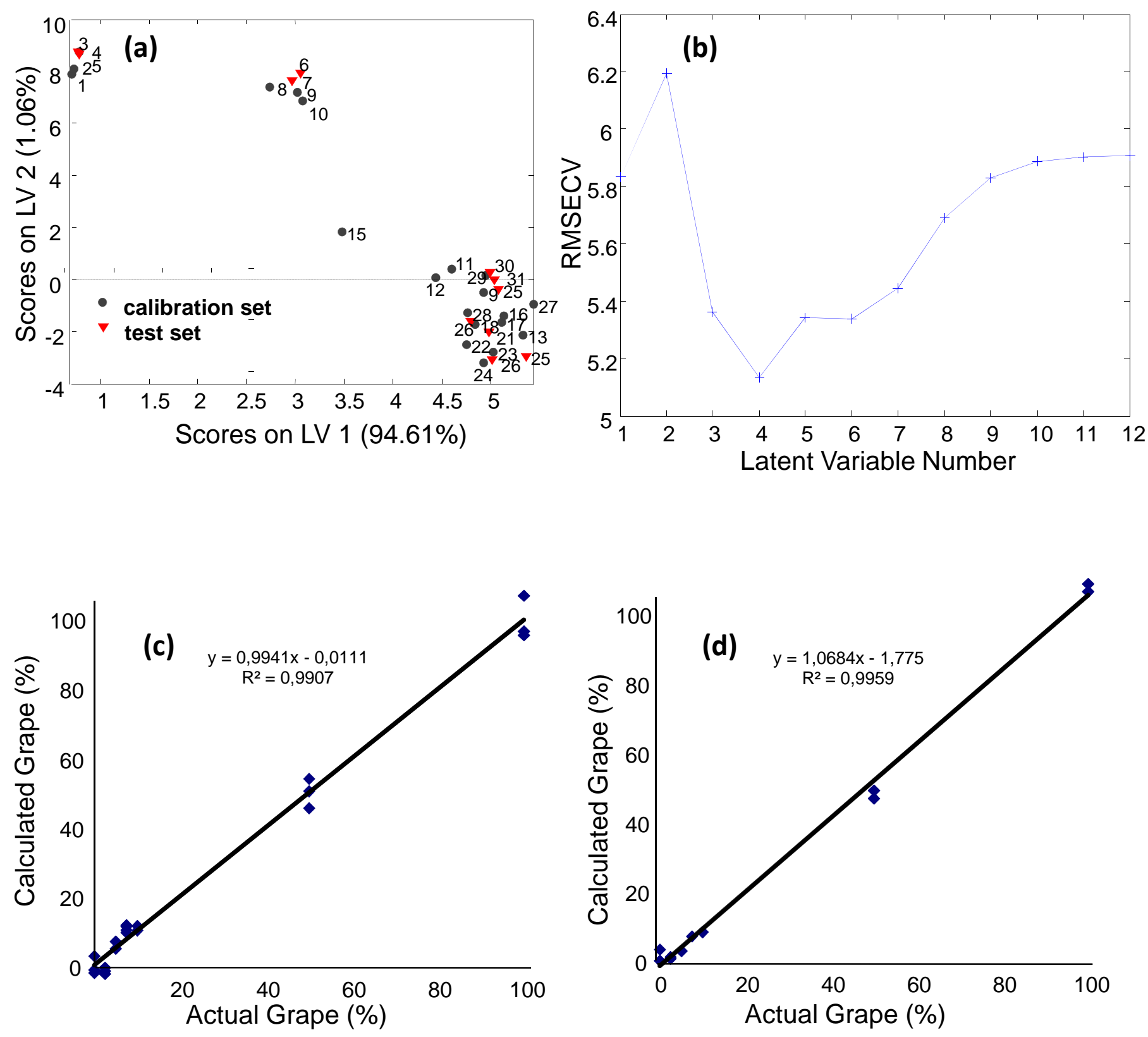